\title{
Catheter-directed interventions in the management of acute pulmonary embolism.
}

\author{
E Skalidis*, I Anastasiou \\ Department of Cardiology, University Hospital of Heraklion, Crete, Greece
}

Accepted on July 29, 2017

Pulmonary embolism (PE) constitutes a leading cause of cardiovascular mortality in the western world, causing 100,000-180,000 deaths annually in the United States [1]. Overall three-month mortality rate in PE patients has been reported to be as high as $15 \%$ in one study [2], but it varies greatly among subsets of patients. High-risk PE patients, presenting with cardiogenic shock or persistent PE-associated hypotension (systolic blood pressure $<90 \mathrm{~mm} \mathrm{Hg}$ or a decline of $>40 \mathrm{~mm} \mathrm{Hg}$ from baseline, persisting for $>15 \mathrm{~min}$ and being not attributable to hypovolemia or sepsis), demonstrate the highest mortality and the immediate administration of direct reperfusion therapy is the mainstay of their management. On the other hand, patients which are hemodynamically stable at presentation, but demonstrate signs of acute right ventricular pressure overload and dysfunction, as assessed by cardiac ultrasound or computed tomography (CT) and/or elevated values of cardiac biomarkers in laboratory testing are considered to belong to the intermediate-risk subset. The Pulmonary Embolism Severity Index (PESI) and the simplified Pulmonary Embolism Severity Index (sPESI) are currently used for further risk stratification of non-high-risk patients. PESI class V patients carry a 30 day of $10.0-24.5 \%$, while the respective value for PESI the relatively lower risk PESI class III patients ranges from $3.2-7.1 \%$. This is comparable to the in-hospital mortality rate of acute coronary syndrome (ACS) patients, and consideration of more advanced treatment modalities than standard systemic anticoagulation alone is thus paramount [3].

An ideal PE reperfusion strategy should be effective in reversing right ventricular dysfunction and reducing adverse clinical events and mortality, without causing a significant increase in the complication rate as compared to treatment with anticoagulation alone.

Systemic thrombolysis, which currently constitutes the most widely advocated reperfusion modality for high-risk PE - in part due to its widespread availability- has been shown to restore pulmonary perfusion more rapidly than systemic anticoagulation alone, leading to hemodynamic improvement and to reversal of right ventricular dilatation and dysfunction [4]. It can also facilitate dissolution of thrombi in the pelvic and lower extremity veins, thereby theoretically decreasing the likelihood of recurrent PE. However, it is associated with rates of major bleeding complications of up to $20 \%$ and intracranial hemorrhage rates of up to $3 \%$ [5]. Although no truly absolute contraindication to thrombolysis exists in the setting of immediately life-threatening acute pulmonary embolism, administration of thrombolytics is strongly discouraged in patients at highest hemorrhagic risk. These include patients with a history of hemorrhagic stroke or stroke of unknown origin at any time, ischemic stroke in the preceding 6 months, central nervous system neoplasm, recent major trauma, surgery or head injury, known bleeding diathesis or gastrointestinal bleeding within the last month [3]. The scenario of a PE patient carrying a high hemorrhagic risk presenting with severe hemodynamic instability is not uncommon; in fact, thrombolysis was withheld in as many as $70 \%$ of hemodynamically unstable - and thus otherwise likely to benefit from direct reperfusion therapy- PE patients in one study [6].

The use of systemic thrombolysis in PE patients at intermediate risk, on the other hand, remains controversial. In the Pulmonary Embolism Thrombolysis (PEITHO) trial, which randomized 1006 hemodynamically stable PE patients with imaging evidence of right ventricular dysfunction and biochemical evidence of myocardial injury to either tenecteplase plus heparin or placebo plus heparin, the primary eficacy outcome - a composite of all-cause death or hemodynamic decompensation within 7 days of randomization - was significantly reduced in the tenecteplase arm. Nevertheless, this reduction was actually driven by a significant reduction of the rate of hemodynamic collapse (not all cause death) and came at the cost of a significant increase in intracranial and non-intracranial major bleeding events [7]. Current guidelines recommend against the routine use of systemic thrombolysis in patients without shock or hypotension, but in parallel the authors state that it should be considered for patients with intermediate-high risk PE and clinical signs of hemodynamic decompensation [3], thus highlighting the need for careful individualization of therapy.

Surgical thromboembolectomy is currently recommended as the reperfusion strategy of choice in the setting of PE with cardiogenic shock or persistent hemodynamic instability and unacceptably high hemorrhagic risk or failed thrombolysis, whereas. According to current guidelines, it may be considered in intermediate-high-risk patients without shock or hypotension if the anticipated risk of bleeding under thrombolytic treatment is high [3]. This treatment modality, however, carries a high mortality rate. In a systematic review, 
mortality was found to be $20 \%$ in patients operated from 1985 to 2005 [8]. In addition, universal surgical expertise is lacking and patient comorbidities commonly contribute to even higher mortality rates.

Due to the aforementioned limitations carried by systemic thrombolytic therapy and surgical thromboembolectomy, clinical interest in the utilization of catheter-directed interventions (CDI) in high-risk and intermediate-risk PE has risen. CDI techniques that are utilized in the management of acute PE can be divided into two categories, with respect to whether local low-dose thrombolysis is administered or not. Techniques that are performed without local thrombolysis - and are thus preferred in patients at highest bleeding risk- include (i) thrombus fragmentation with pigtail or balloon catheters, (ii) rheolytic thrombectomy, (iii) suction embolectomy, (iv) rotational thrombectomy and (v) combined techniques. On the other hand, CDI techniques which utilize local thrombolysis include (i) catheter-directed thrombolysis, (ii) ultrasound-assisted thrombolysis, (iii) pharmacomechanical thrombolysis and (iv) combined techniques.

The most widely used method for pulmonary artery thrombus fragmentation is via manual rotation of a pigtail catheter in the main pulmonary artery or its first order branches. In one study, a recanalization rate of $32.9 \%$ has been reported with this technique in high-risk PE patients [9].

Rheolytic thrombectomy, on the other hand, involves thrombus fragmentation using a saline jet directed from the tip of the catheter with simultaneous emulsified thrombus removal via a separate channel. Available devices include the Amplatz thrombectomy device (Microvena, White Bear Lake, MN, USA), the Cordis hydrolyser hydrodynamics thrombectomy catheter (Cordis, Johnson and Johnson, Japan), the Oasis Thrombectomy System (Boston Scientific Corporation, Natick, MA, USA). The AngioJet Rapid Thrombectomy System, which has been extensively used in the context of percutanecous coronary interventions (PCI), has demonstrated limited efficacy in the treatment of high-risk PE [10,11], whereas several procedure-related complications and deaths prompted the FDA to issue a black-box warning on the device. Several deaths were reported early in the experience of AngioJet thrombectomy for PE, prompting a black-box warning label for its use in the pulmonary arteries by the FDA. Nevertheless, its use is occasionally advocated as a second line option in PE, given prior success.

Earlier suction embolectomy devices, such as the Greenfield - which is no longer commercially availableand the Angiovac devices are hampered by bulky size and difficulty in accessing the pulmonary arteries. On the contrary, modern suction embolectomy catheters such as the Pronto XL extraction catheter (Vascular Solutions, Minneapolis, $\mathrm{MN}$, USA) have been reported to be effective in removing thrombus, reducing mean pulmonary artery pressure and improving hemodynamics in the setting of acute high-risk pulmonary embolism [12].
The efficacy of rotational thrombectomy in patients with high or intermediate-risk PE was tested in one study, in which the Aspirex1 Aspiration Thrombectomy device (Straub Medical, Switzerland) was used. Similar to the AngioJet, the Aspirex1 device employs the Bernoulli hemodynamic principle. A complete thrombus clearance rate $(>90 \%)$ was reported in over $80 \%$ of the 36 treated patients, with concomitant improvement in right heart strain, whereas two major and four minor procedure-related complications were noted [13].

Catheter-directed thrombolysis via multi-side hole infusion catheters is the least technically challenging CDI modality, but its superiority comparatively to systemic thrombolysis with respect to efficacy and/or safety profile has not been proven in large trials. In one randomized controlled study which enrolled high-risk PE patients, there was no difference in angiographically documented posttreatment PE severity between systemic and intrapulmonary administration of $50 \mathrm{mg}$ of alteplase over $2 \mathrm{~h}$ [14].

Ultrasound-assisted catheter-directed thrombolysis (USAT) utilizes high-frequency, low-power ultrasound waves, which cause reversible disaggregation and separation of uncrosslinked fibrin fibers, thereby increasing thrombus permeability for thrombolytic drugs. The ULTIMA trial is one of the three major trials - along with the SEATTLE II trial and the PERFECT prospective registry- which established CDIs as valuable alternatives to other treatment approaches in PE. It this trial, fifty-nine patients with acute main or lower lobe pulmonary embolism and echocardiographic right-toleft-ventricular dimension ratio of at least 1.0 thus regarded as intermediate-risk PE patients were randomized to receive either unfractionated heparin (UFH) and an USAT regimen of $10-20 \mathrm{mg}$ of alteplase over $15 \mathrm{~h}$ or UFH alone. In the USAT arm, significant improvement in the right ventricular parameters was noted, without increased risk of death, major extracranial bleeding or intracranial hemorrhage [15]. Similarly, the SEATTLE II trial, a single arm prospective study of 150 patients with intermediate-risk and high-risk pulmonary embolism, showed a reduction in right ventricular diameter, in the degree of pulmonary hypertension and in anatomic thrombus burden in patients treated with USAT (EkoSonic Endovascular System/EKOS, Bothell, Washington), whereas no patient experienced intracranial hemorrhage [16].

The multicenter Pulmonary Embolism Response to Fragmentation, Embolectomy and Catheter Thrombolysis (PERFECT) registry evaluated various CDI techniques - including catheter-directed mechanical or pharmacomechanical thrombectomy and/or catheter-directed thrombolysis with alteplase or urokinase - in 101 consecutive high-risk and intermediate-risk PE patients. Clinical success was regarded as stabilization of hemodynamics, improvement in pulmonary hypertension, and survival to discharge date. Clinical success rate was $86 \%$ for high-risk and $97 \%$ for intermediate-risk $\mathrm{PE}$, with improvement in pulmonary 
hypertension and RV strain without major hemorrhagic complications [17].

The aforementioned evidence has determined the place of CDI in current guidelines, according to which they should be considered as an alternative to surgical pulmonary embolectomy for PE patients with shock or hypotension in whom full-dose systemic thrombolysis is contraindicated or has failed, whereas they may also be considered as therapeutic strategies in PE patients without shock or hypotension belonging to the intermediate-risk category if the anticipated bleeding risk under thrombolytic treatment is high [3].

Given the limitations carried by thrombolysis and surgical embolectomy as acute reperfusion modalities in high- and intermediate-risk PE, as well as the encouraging results of the three major trials that have evaluated the use of CDI, we feel that the role of CDI will be further upgraded in the future. However, further prospective data from major randomized trials and promotion of universal expertise in the use of these techniques are of utmost importance in this context.

\section{References}

1. Kucher N, Goldhaber SZ. Management of massive pulmonary embolism. Circulation. 2005;112:e28-32.

2. Janata K, Holzer M, Domanovits H, et al. M ortality of patients with pulmonary embolism. Wien Klin Wochenschr. 2002;114(23-24):1026.

3. Konstantinides SV, Torbicki A, Agnelli G, et al. ESC guidelines on the diagnosis and management of acute pulmonary embolism. The Task Force for the diagnosis and management of acute pulmonary embolism of the European Society of Cardiology. Eur Heart J. 2014;35(43):3033-73.

4. Goldhaber SZ, Haire WD, Feldstein ML, et al. Alteplase versus heparin in acute pulmonary embolism: Randomized trial assessing right-ventricular function and pulmonary perfusion. Lancet. 1993;341(8844):507-11.

5. Fiumara K, Kucher N, Fanikos J, et al. Predictors of major hemorrhage following fibrinolysis for acute pulmonary embolism. Am J Cardiol. 2006;97:127-9.

6. Stein PD, Matta F. Thrombolytic therapy in unstable patients with acute pulmonary embolism: Saves lives but underused. Am J Med. 2012;125:465-70.

7. Meyer G, Vicaut E, Danays T, et al. Fibrinolysis for patients with intermediate-risk pulmonary embolism. $\mathrm{N}$ Engl J Med. 2014;370(15):1402-11.

8. Stein PD, Alnas M, Beemath A, et al. Outcome of pulmonary embolectomy. Am J Cardiol. 2007;99(3):421-3.

9. Schmitz-Rode T, Janssens U, Duda SH, et al. Massive pulmonary embolism: Percutaneous emergency treatment by pigtail rotation catheter. J Am Coll Cardiol. 2000;36(2):375-80.
10. Kuo WT, Sze DY, Hofmann LV. Catheter-directed intervention for acute pulmonary embolism: A shining saber. Chest. 2008;133(1):317-8.

11. Kucher N, Windecker S, Banz Y, et al. Percutaneous catheter thrombectomy device for acute pulmonary embolism: In vitro and in vivo testing. Radiology. 2005;236(3):852-8.

12. Heberlein WE, Meek ME, Culp WC, et al. New generation aspiration catheter: Feasibility in the treatment of pulmonary embolism. World J Radiol. 2013;5(11):4305.

13. Dumantepe M, Teymen B, Akturk U, et al. Efficacy of rotational thrombectomy on the mortality of patients with massive and submassive pulmonary embolism. J Card Surg. 2015;30:324-32.

14. Verstraete M, Miller GA, Bournameaux $H$, et al. Intravenous and intrapulmonary recombinant tissue-type plasminogen activator in the treatment of acute massive pulmonary embolism. Circulation. 1988;77:353-60.

15. Kucher N, Boekstegers P, Müller OJ, et al. Randomized, controlled trial of ultrasound-assisted catheter-directed thrombolysis for acute intermediate-risk pulmonary embolism. Circulation. 2014;129(4):479-86.

16. Piazza G, Hohlfelder B, Jaff MR, et al. A prospective, single-arm, multicenter trial of ultrasound-facilitated, catheter-directed, low-dose fibrinolysis for acute massive and submassive pulmonary embolism: The SEATTLE II study. JACC Cardiovasc Interv. 2015;8(10):1382-92.

17. Kuo WT, Banerjee A, Kim PS, et al. Pulmonary embolism response to fragmentation, embolectomy and catheter thrombolysis (PERFECT): Initial results from a prospective multicenter registry. Chest. 2015;148(3):667-73.

\section{*Correspondence to:}

Emmanouil Skalidis

MD, PhD, FESC

Associate Professor of Cardiology

Cardiology Department

University of Crete School of Medicine

University Hospital of Heraklion

Heraklion, Crete

Greece

Tel: +30 2810375261; +306974729223

E-mail: skalides@uoc.gr 